\title{
XVIII CONGRESO INTERNACIONAL DE CIENCIAS ADMINISTRATIVAS
}

$35.001(061.3)$

\author{
por \\ E. Casado Iglesias \\ Profesor de Derecho administrativo \\ de la Universidad Complutense
}

Se ha celebrado en Madrid muy recientemente (en los días 30 de junio al 4 de julio de 1980) el XVIII Congreso Internacional de Ciencias Administrativas.

La celebración del Congreso ha coincidido con el $50^{\circ}$ de la fundación, también precisamente en Madrid, del Instituto Internacional de Ciencias Administrativas, con sede en Bruselas.

Previamente al pleno del Congreso, y con carácter preparatorio del mismo, tuvieron lugar tres sesiones de trabajo (del 26 al 28 de junio) que organizaron, respectivamente, el Instituto Nacional de Administración Pública español, con sede en la antigua Universidad Complutense de Alcalá de Henares, la Generalidad de Cataluña y el Ministerio de Industria, en su sede del Instituto Nacional de Industria.

El primer grupo de trabajo, bajo la presidencia del norteamericano Mr. D. STONE, tuvo como tema «Asociación Internacional de Escuelas e Institutos de Administración»; el celebrado en Bar- 
celona, bajo la presidencia del también norteamericano D. W H ITEHEAD, el de «Administración y Regionalismo», y el celebrado en Madrid, bajo la presidencia del belga Mr. L. PleEbus, se ocupó del concreto punto de «Presupuestos integrados».

El Congreso se celebró bajo el patrocinio de S. M. el Rey de España, D. JUAN CARLos I, y el acto de inauguración, celebrado el día 30 de junio, fue presidido por el Rey, y con él el Presidente actual del Instituto Internacional, Profesor español L. LóPEz RoDo; el Ministro Adjunto al Presidente para la Administración Pública, y también Profesor de Derecho administrativo, S. MARTín-RETORTILlo; el Secretario Adjunto de las Naciones Unidas, Mr. Bi Jilong, y el actual Presidente de la Asociación Española de Ciencias Administrativas, Delegación española del IICA, también Profesor F. GARRIDO FALLA.

El tema general del Congreso fue el de «Respuestas de la Administración pública al reto de las sociedades contemporáneas», y ese temario general fue subdividido en los también cuatro grandes apartados: I. Significado del principio de legalidad en la Administración moderna; II. Problemas del control político sobre los servicios administrativos del Estado y de los Organismos autónomos; III. Características de los funcionarios de la Administración del futuro, y IV. Desarrollo de los métodos y de las técnicas de la planificación en los diferentes sistemas administrativos.

De cada uno de estos cuatro apartados se encargó un Comité, que fue presidido, respectivamente, como relator, el I, por el Juez del Tribunal Supremo Administrativo sueco, Mr. K. Holnagren; el II, por el Director del Real Instituto de Administración Pública Inglesa, Mr. R. NotTAGE; el III, por el Director alemán del Ministerio del Interior, Hans J. von HoERTZEN, y el IV, por el Presidente del Instituto Nacional de Administración Pública mejicano, señor GARCÍA CÁRDENAS.

El primer Comité indicado, y con el título de Derecho y Ciencia de la Administración, tuvo por objeto de estudio los cuatro temas concretos en que fue subdividido aquel contenido más amplio dé «Significado del principio de legalidad en la Administración moderna»: a) Entornos políticos del principio de legalidad, b) Las 
garantías de los derechos individuales, c) Los modelos de adaptación del principio de legalidad a las nuevas misiones de la Administración y $d$ ) Las medidas concretas para asegurar el principio de legalidad, títulos y temas cuyo enunciado y contenido de estudio había sido aprobado por la Tabla Redonda que la Comisión permanente del Instituto Internacional celebró en el año 1979 en Lieja.

El segundo Comité, o de Estructuras Administrativas y Management, lo presidió, como se dijo anteriormente, el inglés Mr. Raymond NotTage, y tuvo como materia más concreta de consideración o estudio los también cuatro temas aprobados en la misma Tabla Redonda de Lieja: $10^{\circ}$ Los principios generales del control político sobre los servicios públicos del Estado y de los Organismos públicos autónomos, $2 .^{\circ}$ ¿Control político o control administrativo?, 3. ${ }^{\circ}$ Proyectos y respuestas, y $4 .^{\circ}$ Control político en los distintos sistemas políticos de la Europa occidental y de la Europa del Este.

El tercer Comité, denominado de Administración del Personal, y con la relatoría del alemán Hans J. von HoERTZEN, examinó los temas más concretos en que fue subdividida su materia: "Características de los funcionarios de la Administración del futuro»: a) Las reformas o innovaciones precisas para la adecuación del personal a las nuevas necesidades de la Administración, b) Formación adecuada de ese personal y c) La función pública internacional del futuro.

El cuarto Comité, con el título de Planificación y Prospectivas, examinó, de acuerdo también con las directrices y temario aprobado en la Tabla Redonda de Lieja referida, los cuatro temas, tan fundamentales en la planificación económica: $10^{\circ} \mathrm{El}$ entorno político, social y cultural de la planificación; $2^{\circ}$ Los aspectos jurídicos de la planificación; $3 .^{\circ}$ Los problemas de realización de los planes económicos y de los políticos, y $4 .^{\circ}$ La prospectiva de la planificación.

Dada la importancia y actualidad en los órdenes internacional y nacionales del tema de la planificación económica y sus diversos aspectos, el anterior temario fue subdividido por el Comité en los otros diversos apartados: a) El personal encargado de la planificación y el nuevo orden económico mundial; b) Democracia y pla- 
nificación; c) Las predicciones del trabajo para los funcionarios internacionales y nacionales; d) La planificación social; e) Las relaciones entre las autoridades planificadoras y las instituciones financieras; f) Información y planificación; $g$ ) Las relaciones entre las autoridades planificadoras y los Ministerios; $h$ ) Información y planificación; i) El contrato como instrumento o sistema de planificación para la implantación de planes y programas; j) La cooperación internacional en la planificación y la prospectiva.

Algunos de estos temas, en cuyo esclarecimiento y debate participó el autor, habían sido ya parcialmente enunciados en la reunión del Instituto Internacional de Méjico e, igualmente, examinados por el Grupo de Administración Pública del Instituto Internacional de Administración Pública en su reunión europea de París, 1978, y el Comité de «Planificación y Prospectivas» de este XVIII Congreso Internacional de Ciencias Administrativas los compendió y examinó en la forma relacionada anteriormente.

Al Congreso asistieron representantes de diversos Organismos internacionales: Banco Interamericano de Desarrollo, Banco Mundial, Centro Africano de Formación y Recursos Administrativos para el Desarrollo, Comisión de las Comunidades Europeas, Comisión Económica para Europa, Comisión Económica para América Latina, Consejo de Europa, Naciones Unidas, Organización Arabe de Ciencias Administrativas, Organización de Cooperación y de Desarrollo Económico, Programa de las Naciones Unidas para el Desarrollo, Unión Internacional de Ciudades y Poderes Locales, así como representantes o delegados de setenta y ocho países de los más diversos continentes, que totalizaron la cifra de mil doscientos delegados, entre los que se encontraban más de treinta y cinco Ministros de las diversas ramas administrativas de sus respectivos países.

España colaboró en el Congreso con numerosos Profesores universitarios, altos funcionarios de la Administración y de otros Organismos y la participación especial de la ya citada Asociación Española de Ciencias Administrativas, Delegación española del IICA.

Las numerosas ponencias presentadas, con la diversidad de los temas examinados por el Congreso, hacen imposible una cita, ni siquiera parcial. 
Ello no obstante, hemos de destacar, por nuestra parte, las presentadas por los españoles BAENA DEL ALCÁZAR, ARIÑo ORTIZ, BAÑóN MARTínez y M. DEL Pozo sobre el problema del control político de los servicios del Estado, y las presentadas sobre el mismo tema por los Profesores francés, L. LESAGE; alemán, G. W. WILTKÄMBER, y norteamericano, Geral E. LEIDEN.

El Profesor Baena del Alcázar puso, en su ponencia, muy expresamente de manifiesto que el gran número de Organismos cuasiautónomos y su crecimiento cuantitativo se debía a la imposibilidad de prestar el Estado todas las necesidades de la sociedad actual.

Más pesimista en su comunicación, el Profesor ARIÑo ORTIZ. manifestó las grandes dificultades o casi imposibilidad de un real equilibrio entre los poderes estatales y las empresas públicas.

BAÑón Martínez, en ponencia referida más concretamente a nuestro país, resaltó que el control estatal en estos Organismos. autónomos hay que considerarlo como un problema de control común o general a todas las Administraciones contemporáneas, manifestándose partidario, más que de un control político, de un. control social.

El Profesor Molina DEL Pozo, en también comunicación referida concretamente a la reorganización de la Administración central española llevada a cabo en 1977, manifestó no encontrarla: adecuada ni clara, por cuanto en ella no quedan bien definidas las diversas competencias ministeriales ni la responsabilidad de ese control central en los diversos o concretos Ministerios.

También nos merecen cita especial las comunicaciones presentadas, en relación al tema de la planificación, por el Director del Centro de Estudios Argentino, O. OlzlaK, y las de los Profesores L. Nizaro (francés); el de la Costa de Marfil, N’Dri KPAPCHibo, y la del Director de Administración y Finanzas mejicano, CAMARENA: ADAME, dando a conocer, detalladamente, las directrices, contenido y logros del último plan nacional de desarrollo de su país, de 1978.

La publicación por el Instituto Internacional de un número de su Revista Internacional de Ciencias Administrativas dedicado especialmente a España, y en el que han colaborado prestigiosas firmas de la ciencia administrativa española, completaron, con otros actos y recepciones que diversos Organismos españoles ofrecieron a los 
congresistas, el apretado programa de este XVIII Congreso Internacional de Ciencias Administrativas, que ha servido, aparte las numerosas tentativas de resolver los problemas de las Administraciones actuales, para poner en contacto a los asistentes de tan diversos países concurrentes y la posibilidad de poder contrastar puntos de vista nacionales en los tan complejos temas y problemas en que se debaten estas Administraciones contemporáneas. 